\title{
Influence of diabetes mellitus on the heart and macrovascular mortality
}

\author{
E . Standl, O. Schnell, B . B alletshofer, E . Schleicher, D . M uhr, A . G . Z iegler, M . H aslbeck
}

Institute of Diabetes Research, Munich, Germany

This report deals with studies on

a) molecular effects of glucose on large vessel cells and the related extracellular matrix;

b) the predictive power of long-term blood glucose control on macrovascular mortality of NIDDM patients; and

c) early cardiac sympathetic dysinnervation in subjects with IDDM.

The excess of glucose appears to play an important and specific role in the genesis of macroangiopathy in diabetic patients. Activation of protein kinase $\mathrm{C}$, the sorbitol pathway, and advanced glycation end-product formation are thought to be the major pathways linking the degree of glycaemic compensation with the pathogenetic process of macrovascular disease. In particular, glucose-induced activation of protein kinase $\mathrm{C}$ seems to be a key event in how hyperglycaemia exerts its effect on endothelial, myomedial and mesangial cells. In cultured endothelial cells from porcine aorta, acute stimulation of protein kinase $\mathrm{C}$ was found to lead to an increased synthesis of heparan sulphate proteoglycan (HSPG), whereas chronic stimulation generated a $50 \%$ drop of HSPG synthesis. The mRNA for HSPG was also shown to be decreased. A decreased formation of HSPG by endothelial cells of large arteries exposed to chronic hyperglycaemia seems to be relevant also for the in vivo situation of human diabetes, since a significant reduction of HSPG has been described in coronary arteries of non-insulin-dependent diabetic (NIDDM) patients and a decreased ratio of heparan sulphate/ dermatan sulphate in the intima layer of aortas from

Corresponding author: Professor E. Standl, Forschergruppe Diabetes, III Medizinische Abteilung, Krankenhaus Schwabing, Kölner Platz 1, D-80804 München, Germany

Abbreviations: HSPG, Heparan sulphate proteoglycan; NIDDM, non-insulin-dependent diabetes mellitus; ${ }^{123} \mathrm{~J}$ MIBG, ${ }^{123} \mathrm{~J}$-metaiodobenzylguanidine. diabetic patients was most pronounced in (diabetic) plaques. At the same time, chronic activation of protein kinase $\mathrm{C}$ is also associated with a profound change of the extracellular matrix of the heart in terms of an increased synthesis of collagen IV and VI.

Against this background, we performed a 10-year follow-up of the Munich General Practitioner Project, to evaluate the long-term effects of many of the presently discussed cardiovascular risk factors, including parameters of blood glucose control, on the outcome in terms of macrovascular and overall mortality in a random cohort of NIDDM patients. Of the original 290 patients (103 males, 187 females, median age 65 years) $92.5 \%$ could be assessed; 103 subjects had died, 58 from macrovascular causes. In a univariate analysis of baseline data, deceased patients, and especially those who died from macrovascular causes, had significantly higher fasting blood glucose, $\mathrm{HbA}_{1 \mathrm{c}}$, von Willebrand-factor protein, urine albumin excretion, and serum $\beta 2$-microglobulin, were significantly older, exhibited significantly more ischaemic heart disease (abnormal ECG Minnesota codes), carotid artery and peripheral vascular disease (both determined by ultrasound-Doppler), and had significantly inferior knowledge about diabetes and its treatment. No significant differences were seen for gender, blood pressure, smoking, total cholesterol, triglycerides, HDL-cholesterol, or the use of antidiabetic, antihypertensive or coronary drugs. In a multiple logistic regression analysis, the risk factors for macrovascular death were age, $\mathrm{HbA}_{1 \mathrm{c}}$ and von Willebrand-factor protein. When baseline macrovascular disease was taken into account, carotid artery disease was also a determinant. The main variables for the metabolic syndrome (blood pressure, dyslipidaemia, body mass index) did not enter a multiple logistic regression analysis except $\mathrm{HbA}_{1 \mathrm{c}}$. The data suggest that age and haemoglobin $A_{1 c}$ are major determinants, and that in addition von Willebrand-factor associated 
endothelial damage is a risk factor for macrovascular mortality in NIDDM patients. Recent studies have indicated that the release of von Willebrand-factor protein is closely related to vascular endothelial growth factor and also insulin resistance.

Using the innovative technique of scintigraphic detection with ${ }^{123} \mathrm{~J}$-MIBG $\left({ }^{123} \mathrm{~J}\right.$-metaiodobenzylguanidine) cardiac sympathetic integrity was studied both in newly manifest and long-term subjects with IDDM. This technique seems to be far more sensitive than the usual ECG-based tests for assessing cardiac autonomic neuropathy. With this approach 17 (77\%) out of 22 newly diagnosed, yet metabolically stabilized IDDM patients (without myocardial perfusion abnormalities) exhibited signs of sympathetic dysinnervation, especially in the posterior myocardial region. In contrast, only two of these diabetic patients $(9 \%)$ showed an ECG-based cardiac autonomic neuropathy $(2$ or more of 5 age-related cardiac reflex tests abnormal). A 1-year follow-up study indicated partial restoration of this impairment by good metabolic control. The global myocardial ${ }^{123} \mathrm{~J}$-MIBG had improved in 7 patients, remained unchanged in another 7 , and deteriorated in 2 patients at 1-year follow-up. It is suggested that even in the early stage of IDDM, cardiac sympathetic dysinnervation is composed of reversible and irreversible neuronal abnormalities.

In long-term IDDM patients, however, sympathetic dysinnervation was found independent of current $\mathrm{HbA}_{1 \mathrm{c}}$ levels, in many patients (16 out of 22 ) even without ECG-based autonomic neuropathy, again especially in the posterior wall. In addition, all 20 of the long-term patients with 2 or more abnormal ECG reflex tests indicating autonomic neuropathy also showed a severely disordered ${ }^{123}$ J-MIBG pattern on scintigraphic assessment. So far, very little improvement of the scintigraphic abnormalities was found in an ongoing 2-year follow-up of the longterm patients, despite a reasonably good control close to normoglycaemia in most patients. This seemingly partial independence of cardiac sympathetic impairment from metabolic parameters may perhaps be seen in the context of another intriguing finding, i.e. the presence of autoantibodies against sympathetic ganglia in newly diagnosed and long-term IDDM patients. These autoantibodies were exhibited in $40 \%$ of newly diagnosed and $25 \%$ of long-term IDDM patients. Furthermore, these antibodies correlated closely with a reduction of the global myocardial uptake of ${ }^{123} \mathrm{~J}$-MIBG. At present, the role of autoantibodies against parasympathetic tissues is under investigation.

The overall importance of these MIBG-changes in IDDM patients may become apparent against the background of observations in the context of the familial long QT syndrome, which is totally independent from diabetes, but shows similarly disordered ${ }^{123} \mathrm{~J}-\mathrm{MIBG}$ patterns and is almost invariably burdened with the problem of sudden death. 